\title{
ZEROS OF SELF-INVERSIVE POLYNOMIALS
}

\author{
F. F. BONSALL AND MORRIS MARDEN
}

1. Introduction. In the present paper we are concerned with polynomials $g(z)=b_{0}+b_{1} z+\cdots+b_{m} z^{m}$ (in the complex variable $z$ ) whose zeros are symmetric in the unit circle $C:|z|=1$. Since these zeros form a set of numbers which is invariant under the transformation $w=1 / \bar{z}$ of inversion in $C$, we shall refer to such $g(z)$ as self-inversive polynomials.

Our present interest in self-inversive polynomials pertains to the Schur-Cohn-Marden [2] method for enumerating the zeros of a polynomial $f(z)=a_{0}+a_{1} z+\cdots+a_{n} z^{n}$ within $C$. This method consists in defining the polynomial

$$
f^{*}(z)=\bar{a}_{n}+\bar{a}_{n-1} z+\cdots+\bar{a}_{0} z^{n}
$$

and introducing the sequence of polynomials

$$
f_{j}(z)=a_{0}^{(j)}+a_{1}^{(j)} z+\cdots+a_{n-j}^{(j)} z^{n-j}
$$

by the relations

$$
f_{0}(z)=f(z), \quad f_{j+1}(z)=\bar{a}_{0}^{(j)} f_{j}(z)-a_{n-j}^{(j)} f_{j}^{*}(z)
$$

for $j=1,2, \cdots, n$. Then, if $p$ of the products

$$
P_{k}=a_{0}^{(1)} a_{0}^{(2)} \cdots a_{0}^{(k)}, \quad k=1,2, \cdots, n,
$$

are negative and if the remaining $n-p$ are positive, $f(z)$ has exactly $p$ zeros inside $C$.

This method, however, does not apply whenever $a_{0}^{(k)}=0$ for some $k, 1 \leqq k \leqq n$. Thus, in particular, it does not apply whenever, for some such $k, f_{k-1}(z)$ is self-inversive. In such a case $f_{k}(z)$, which is identically zero, must be replaced in the sequence (1.1) by the polynomial $\left[f_{k-1}^{\prime}(z)\right]^{*}$ in accordance with the following theorem due to Cohn [1].

THEOREM I. Let $g(z)=b_{0}+b_{1} z+\cdots+b_{m} z^{m}$ be a self-inversive polynomial; that is, let

$$
b_{m}=u \bar{b}_{0}, b_{m-1}=u \bar{b}_{1}, \cdots, b_{0}=u \bar{b}_{m}, \quad|u|=1 .
$$

Then $g(z)$ has the same number of zeros inside the unit circle $C:|z|=1$ as does the polynomial

Presented to the Society, December 28, 1950; received by the editors July 17, 1951 and, in revised form, November 16, 1951. 
(1.3) $G(z)=\left[g^{\prime}(z)\right]^{*}=m b_{m}+(m-1) b_{m-1} z+\cdots+b_{1} z^{m-1}$.

That is, $g(z)$ and its derivative $g^{\prime}(z)$ have same number of zeros in the closed exterior of $C$.

The primary objective of the present note is to furnish for this theorem a new proof which is simpler than Cohn's and which makes the substitution of $\left[f_{j}^{\prime}(z)\right]^{*}$ for $f_{j+1}(z)$ seem more natural.

2. A lemma. For the proof of the theorem the following lemma will be needed.

LEMмA. If $g(z)$ is a self-inversive polynomial, the only zeros of its first derivative $g^{\prime}(z)$ on the unit circle $|z|=1$ are at the multiple zeros of $g(z)$ on $|z|=1$.

To establish this lemma, it suffices to show that, if $g(1) \neq 0$, then $g^{\prime}(1) \neq 0$.

Let $g(z)=\prod_{1}^{m}\left(z-z_{j}\right)$. Then

$$
g^{\prime}(1) / g(1)=\sum_{1}^{m} w_{j}, \quad w_{j}=\left(1-z_{j}\right)^{-1} .
$$

But the transformation $w=(1-z)^{-1}$ with $w=u+i v$ carries the circle $|z|=1$ into the straight line $u=1 / 2$ and the points $z_{j}$ into points $w_{j}$ symmetric in this line. Since also the center of gravity $W$ of the $w_{j}$ lies on this line, $W=m^{-1} \sum_{1}^{m} w_{j} \neq 0$. That is, $g^{\prime}(1) \neq 0$.

3. Proof of the theorem. Let the polynomial $f_{1}(z)$ be formed for

$$
f(z)=g(r z)=\sum_{0}^{m}\left(b_{k} r^{k}\right) z^{k}, \quad 0<r<1,
$$

in accordance with (1.1). Thus,

$$
f_{1}(z)=b_{0} f(z)-b_{m} r^{m} f^{*}(z) .
$$

In view of (1.2),

$$
f_{1}(z)=\sum_{k=0}^{m-1} b_{m} b_{m-k} r^{k}\left[1-r^{2(m-k)}\right] z^{k}
$$

Let $p$ denote the number of zeros of $g(z)$ in $|z|<1$. Then, if $r$ is sufficiently close to one, $f(z)$ will also have $p$ zeros in $|z|<1$. Furthermore, since $\left|f^{*}\left(e^{i \theta}\right)\right|=\left|f\left(e^{i \theta}\right)\right|$ and hence

$$
\left|b_{m} r^{m} f^{*}\left(e^{i \theta}\right)\right|<\left|b_{0} f\left(e^{i \theta}\right)\right|
$$

it follows from Rouché's Theorem that $f_{1}(z)$ has also $p$ zeros in $|z|<1$. 
Now, let

$$
F_{1}(z)=f_{1}(z) /\left[b_{m}\left(1-r^{2}\right)\right] .
$$

Clearly, $F_{1}(z)$ has also $p$ zeros in $|z|<1$. In view of (3.3) and (1.3), as $r \uparrow 1$,

$$
\lim F_{1}(z)=\sum_{0}^{m-1}(m-k) b_{m-k} z^{k}=G(z) .
$$

Since this convergence is uniform in $|z| \leqq 1$, it follows from Hurwitz' theorem that every zero $\zeta$ of $G(z),|\zeta|<1$, with a multiplicity $\mu$, is the limit of zeros, in $|z|<1$, of $F_{1}(z)$ with a total multiplicity of $\mu$. Hence, there are at most $p$ zeros of $G(z)$ in $|z|<1$, and at least $p$ zeros of $G(z)$ in $|z| \leqq 1$.

There are now three cases to consider.

Case I. $g(z)$ has no zeros on $|z|=1$. According to the lemma, $g^{\prime}(z)$ $\neq 0$ and hence $G(z) \neq 0$ on $|z|=1$. It follows that no zero of $F_{1}(z)$ can approach the circumference of the unit circle as $r \uparrow 1$, and therefore $G(z)$ has exactly $p$ zeros in $|z|<1$.

Case II. $g(z)$ has on $|z|=1$ only zeros of even multiplicity. Let these zeros be denoted by $e^{i \theta_{k}}$ and their multiplicities by $2 s_{k}, k$ $=1,2, \cdots, q$. With $0<r<1$, let

$$
h(z)=g(z) \prod_{k=1}^{q} \frac{\left(z-r e^{\theta_{k i}}\right)^{s_{k}}\left(z-r^{-1} e^{\theta_{k i}}\right)^{s_{k}}}{\left(z-e^{\theta_{k} i}\right)^{2 s k}},
$$

and $H(z)=\left[h^{\prime}(z)\right]^{*}$. Then, since $h(z)$ is a polynomial belonging to case $I, H(z)$ has $p+\sigma$ zeros in $|z|<1$, where $\sigma=\sum_{1}^{e} s_{k}$. Since each $\left(2 s_{k}-1\right)$-fold zero $e^{i \theta_{k}}$ of $G(z)$ is approached as $r \uparrow 1$ by the $\left(s_{k}-1\right)$ fold zeros $r^{ \pm 1} e^{i \theta_{k}}$ of $H(z)$, at most one additional zero of $H(z)$ can approach $e^{i \theta_{k}}$ from the inside of circle $|z|=1$. Hence, at most $\sigma$ zeros of $H(z)$ approach points on the boundary from inside $|z|=1$. That is, at least $p$ zeros of $G(z)$ are in $|z|<1$. Since it is already known that at most $p$ zeros of $G(z)$ lie in $|z|<1$, there are exactly $p$ zeros of $G(z)$ in $|z|<1$.

Case III. $g(z)$ has on $|z|=1$ at least one zero of odd multiplicity. In this case, let $\phi(z)=[g(z)]^{2}$. Since $\phi(z)$ is a polynomial belonging to Case II with $2 p$ zeros in $|z|<1$, then $\left[\phi^{\prime}(z)\right]^{*}$ has also $2 p$ zeros in $|z|<1$. But $\left[\phi^{\prime}(z)\right]^{*}=2 g^{*}(z) G(z)$. Since $g^{*}(z)$ has $p$ zeros in $|z|<1$, it follows then that $G(z)$ has exactly $p$ zeros in $|z|<1$. This completes the proof of Theorem I.

4. Polar derivative of a real polynomial. Theorem I and the lemma will now be used to establish the following result. 
THEOREM II. Let $f(w)$, a real polynomial of degree $m$, have exactly $p$ zeros in the upper half-plane $J(w)>0$ and let $\zeta$ be any point not on the real axis and not a zero of $f(w)$. Then the polar derivative

$$
F(w)=(w-\zeta) f^{\prime}(w)-m f(w)
$$

of $f(w)$ with pole at $\zeta$ has exactly $p$ zeros in the half-plane $J(w) J(\zeta)>0$ and its only real zeros are at the multiple real zeros, if any, of $f(w)$.

To prove this theorem, let transformation $z=(w-\bar{\zeta}) /(w-\zeta)$ be introduced. This maps the half-plane $J(w) J(\zeta)<0$ upon the interior of the unit circle in the $z$-plane. Let

$$
\begin{aligned}
g(z) & =(z-1)^{m} f((\zeta z-\bar{\zeta}) /(z-1)), \\
\Gamma(z) & =(z-1)^{m-1} F((\zeta z-\bar{\zeta}) /(z-1)) .
\end{aligned}
$$

Then according to (4.1) and (4.2),

$$
\begin{aligned}
& \Gamma(z)=(z-1)^{m-1}\left\{\frac{\zeta-\bar{\zeta}}{z-1} f^{\prime}\left(\frac{\zeta z-\bar{\zeta}}{z-1}\right)-m f\left(\frac{\zeta z-\bar{\zeta}}{z-1}\right)\right\}, \\
& g^{\prime}(z)=m(z-1)^{m-1} f\left(\frac{\zeta z-\bar{\zeta}}{z-1}\right)+(z-1)^{m-1} f^{\prime}\left(\frac{\zeta z-\bar{\zeta}}{z-1}\right)\left(\frac{\xi-\zeta}{z-1}\right) .
\end{aligned}
$$

That is, $\Gamma(z)=-g^{\prime}(z)$.

Since $g(z)$ is a self-inversive polynomial with $p$ zeros in the circle $C:|z|<1, \Gamma^{*}(z)$ has $p$ zeros inside $C$ and therefore $\Gamma(z)$ has $p$ zeros outside $C$. Hence, $F(w)$ has $p$ zeros in the half-plane $J(w) J(\zeta)>0$.

The remaining conclusion of Theorem II follows immediately from the lemma.

5. Alternative proof and corollary. Professor J. L. Walsh has very kindly called the attention of the authors to the possibility of basing a proof of Theorem I upon the Principle of Argument, essentially as follows:

Let us write $\gamma_{j}=\alpha_{j}+i \beta_{j}, \gamma_{j}^{*}=1 / \bar{\gamma}_{j}$ when $\left|\gamma_{j}\right| \neq 1, \delta_{j}=e^{i \theta i}$, and

$$
g(z)=b_{m} \prod_{j=1}^{p}\left(z-\gamma_{j}\right)\left(z-\gamma_{j}^{*}\right) \prod_{j=1}^{q}\left(z-\delta_{j}\right) .
$$

Let $C^{\prime}$ be the smallest closed curve which contains the configuration consisting of circle $C:|z|=1$ and circles of radius $\epsilon$ about each $\delta_{j}$, where $\epsilon$ is a sufficiently small positive number. It may be then shown that the vector $v(z)=-g^{\prime}(z) / g(z)$ has an inwardly pointing, radial component at each point $z$ of $C^{\prime}$ and hence that the angle of vector $v(z)$ decreases by $2 \pi$ as $z$ traverses $C^{\prime}$ once counterclockwise. 
Theorem I then follows at once.

As indicated by Walsh, this method of proof leads also to the following conclusion [3]. Let the (Jensen) circles $J_{k}$ be drawn having as diameters the lines joining the pairs of zeros $\gamma_{k}, \gamma_{k}^{*}, k=1,2, \cdots, p$; then any configuration consisting of the interiors of certain $J_{k}$ but disjoint from the remaining $J_{k}$ contains exterior to $C$ the same number of zeros of $g(z)$ and $g^{\prime}(z)$.

The authors have also developed some additional proofs based upon certain more general investigations which will be published at a later date.

\section{BIBLIOGRAPHY}

1. A. Cohn, Über die Anzahl der Wurzeln einer Algebraischen Gleichung in einer Kreise, Math. Zeit. vol. 14 (1922) pp. 110-148.

2. M. Marden, The geometry of the zeros of a polynomial in a complex variable, Mathematical Surveys, no. 3, New York, American Mathematical Society, 1949; see p. 150.

3. J. L. Walsh, Location of the critical points, Amer. Math. Soc. Colloquium Publications, vol. 34, 1950, pp. 52-55.

UNIVERSITY OF DURHAM AND

University of Wisconsin, MilwaUkeE 